\title{
Is PTEN loss associated with clinical outcome measures in human prostate cancer?
}

\author{
P McCall', CJ Witton ${ }^{2,3}$, S Grimsley', KV Nielsen ${ }^{2}$ and J Edwards",I \\ 'Section of Surgery, Division of Cancer Sciences and Molecular Pathology, University of Glasgow, Glasgow Royal Infirmary, Glasgow G3I 3ER, UK; \\ ${ }^{2}$ Department of Molecular Pathology, Dako A/S, Glostrup, Denmark
}

Inactivating PTEN mutations are commonly found in prostate cancer, resulting in an increased activation of Akt. In this study, we investigate the role of PTEN deletion and protein expression in the development of hormone-refractory prostate cancer using matched hormone-sensitive and hormone-refractory tumours. Fluorescent in situ hybridisation and immunohistochemistry was carried out to investigate PTEN gene deletion and PTEN protein expression in the transition from hormone-sensitive to hormonerefractory prostate cancer utilising 68 matched hormone sensitive and hormone-refractory tumour pairs (one before and one after hormone relapse). Heterogeneous PTEN gene deletion was observed in $23 \%$ of hormone sensitive tumours. This increased significantly to $52 \%$ in hormone-refractory tumours $(P=0.044)$. PTEN protein expression was observed in the membrane, cytoplasm and the nucleus. In hormone sensitive tumours, low levels of cytoplasmic PTEN was independently associated with shorter time to relapse compared to high levels of PTEN $(P=0.028$, hazard ratio $0.5 \mathrm{I}(95 \% \mathrm{Cl} 0.27-0.93)$. Loss of PTEN expression in the nucleus of hormone sensitive tumours was independently associated with disease-specific survival $(P=0.031$, hazard ratio $0.52,95 \% \mathrm{Cl}$ $0.29-0.95)$. The results from this study demonstrate a role for both cytoplasmic and nuclear PTEN in progression of prostate cancer to the hormone-refractory state.

British Journal of Cancer (2008) 99, 1296-1301. doi:I0.1038/sj.bjc.6604680 www.bjcancer.com

(C) 2008 Cancer Research UK

Keywords: PTEN; Akt; prostate; hormone refractory

Every year in the Unite Kingdom, almost 32000 cases of prostate cancer are diagnosed and approximately 10000 die of the disease (Cancer Research UK web site, 2004). The underlying molecular mechanisms of prostate cancer initiation and progression are largely unknown because of extensive tumour heterogeneity. Patients diagnosed with locally advanced or metastatic prostate cancer may be treated by androgen ablation therapy, resulting in a reduction of androgens in the circulation and inhibition of tumour growth. This treatment is initially successful, but patients tend to relapse within 18-24 months with disease progression refractory to therapy (Arnold and Isaacs, 2002). Hormone-refractory prostate cancer has a poor prognosis, with median survival period reported to be 24 months. Loss of androgen dependence is often correlated with the overexpression of antiapoptotic and cell survival signalling pathways (Johnson and Hamdy, 1998; Karan et al, 2003). Components of these pathways are mutated or abnormally expressed in many tumour types and mutations of upstream receptors, such as EGFR, transmit their signals through these

*Correspondence: Dr J Edwards, Department of Surgery, University Of Glasgow, Division of Cancer Sciences and Molecular Pathology, Level 2, Queen Elizabeth Building, Glasgow Royal Infirmary, Glasgow G3I 2ER, UK; E-mail: jelOb@clinmed.gla.ac.uk

${ }^{3}$ Current address: LiPlasome Pharma A/S, Danish Technical University, Lyngby, Denmark

Received 10 July 2008; revised 27 August 2008; accepted 27 August 2008 cascades. The loss of tumour suppressor function is also a common event in the progression to hormone-refractory prostate cancer and is associated with the gain of oncogenic signalling (Isaacs and Kainu, 2001). P53, retinablastoma (Rb), NKX3.1 and phosphatase and tensin homologue deleted on chromosome 10 (PTEN) have all been well documented to have a loss of function during the progression to hormone-refractory prostate cancer (Bookstein et al, 1990; Phillips et al, 1994; He et al, 1997; Davies et al, 1999; McMenamin et al, 1999).

Phosphatase and tensin homologue deleted on chromosome 10 functions as a lipid phosphatase that dephosporylates phosphatidylinositol (3,4 and 5) triphosphate (PIP3), a second messenger of PI3K at the plasma membrane. PIP3 recruits phosphatidylinositol-dependent kinase-1 (PDK1) and Akt to the membrane. Here, PDK1 phosphorylates Akt, which leads to its activation. Akt mediates signals downstream that promote cell survival and proliferation. Phosphatase and tensin homologue deleted on chromosome 10 mutants that retain protein tyrosine phosphatase activity, but lose the ability to dephosphorylate PIP3, are found in many tumours, indicating that the lipid phosphatase activity is needed for tumour suppression (Myers et al, 1998). To function in this manner, PTEN must be located in the cytoplasm; however, it has recently emerged that there is also a role for PTEN in the nucleus (Eng, 2003; Chung and Eng, 2005; Lian and Di Cristofano, 2005).

Prostate cancer cell lines that have been cultured from metastatic sites, such as the lymph nodes (LNCaP) or brain metastasis (PC3) have highly active PI3K/Akt signalling (Davies 
et al, 1999; Murillo et al, 2001). PTEN mutation has been associated with $5-27 \%$ of localised and $30-60 \%$ of metastatic prostate tumours (Feilotter et al, 1998; Suzuki et al, 1998; Wang et al, 1998). In addition, loss of PTEN expression is associated with disease progression and increased risk of recurrence (Gray et al, 1998; McMenamin et al, 1999; Burton et al, 2000; Reiss et al, 2000; Fenci and Woenckhaus, 2002), although substantial heterogeneity has been observed between different metastatic sites within the same patients (Suzuki et al, 1998). Here, we examined both the deletion of the PTEN gene and expression of PTEN protein at individual cellular locations in a cohort of matched hormone-sensitive and hormone-refractory prostate tumours, with the aim of clarifying the prognostic significance of PTEN loss in prostate cancer. Using the same cohort, we have previously demonstrated that the upregulation of Akt activation is significantly associated with decreased survival, and therefore PTEN loss is one possible route by which this may occur (McCall et al, 2008).

\section{MATERIALS AND METHODS}

\section{Patients}

Sixty-eight patients with matched hormone-sensitive and hormone-refractory tumour pairs were retrospectively selected for the analysis. All tumours had patient identification removed, and the clinical information database was anonymised. Ethical approval was obtained from the Multicentre Research Ethics Committee for Scotland (MREC/01/0/36) and fron the Local Research and Ethical Committees. Patients were only selected for analysis if they initially responded to hormone treatment (in the form of subcapsular bilateral orchidectomy or maximum androgen blockade), but subsequently relapsed (two consecutive rises in PSA greater than 10\%) and had a pre- and post-hormone relapse tissue sample available for analysis. Hormone-refractory prostate cancer specimens were obtained by TURP, when patients required further surgical procedures to treat clinical symptoms such as bladder outflow obstruction. All these samples were obtained with 8 weeks of biochemical relapse being diagnosed (two consecutive rises in PSA greater than 10\%). Phosphorylated Akt expression is already available for this patient cohort (McCall et al, 2008).

\section{Fluorescent in situ hybridisation}

Fluorescent in situ hybridisation (FISH) was performed on $5 \mu \mathrm{m}$, archival formalin fixed, paraffin-embedded prostate tumour tissue arrays. Slides were incubated for $1 \mathrm{~h}$ at $56^{\circ} \mathrm{C}$, dewaxed and rehydrated through graded alcohols. Tissue was then pretreated using histology FISH accessory kit (Dako A/S, Glostrup, Denmark). In brief, slides were rinsed twice in wash buffer and then incubated for $10 \mathrm{~min}$ in pretreatment buffer at $95^{\circ} \mathrm{C}$, followed by a 3-min incubation at room temperature in wash buffer. Slides were then incubated in pepsin for $26 \mathrm{~min}$ at $25^{\circ} \mathrm{C}$, followed by a 3-min incubation at room temperature in wash buffer. Dako A/S provided PTEN probe; $10 \mu \mathrm{l}$ of probe was applied to each slide and incubated at $82^{\circ} \mathrm{C}$ for $22 \mathrm{~min}$ followed by an overnight incubation at $45^{\circ} \mathrm{C}$. Slides were then washed in stringent wash buffer for $10 \mathrm{~min}$ at $65^{\circ} \mathrm{C}$, followed by two 3-min washes in wash buffer. Slides were dehydrated through graded alcohols, mounted in DAPI Vectashield (Vector Laboratories, CA, USA) and viewed using a Leica DMLB microscope at $\times 400$ magnification (Figure 1A). Fluorescent in situ hybridisation was scored by two independent observers; the number of signals for each chromosome (green) and gene (red) were counted in 20 nonoverlapping nuclei. The gene-to-chromosome ratio was then calculated. PTEN loss was classified as a gene-to-chromosome ratio of $\leqslant 0.8$ (Watters et al, 1998).

\section{Immunohistochemistry}

Tumour expression of PTEN was determined in archival formalin fixed, paraffin-embedded prostate tissue sections $(5 \mu \mathrm{m})$ by IHC. Sections were dewaxed in xylene and rehydrated through graded alcohols before blocking endogenous peroxidase in 3\% hydrogen peroxide. Antigen retrieval was performed by heating tissue sections under pressure in citrate buffer $(\mathrm{pH} 6)$ for 5 min. Sections were blocked using $1.5 \%$ horse serum; PTEN antibody (Cell Signalling Technology) was used at $1 \mu \mathrm{g} \mathrm{ml}^{-1}$, and incubated overnight at $4{ }^{\circ} \mathrm{C}$. Staining was developed using Envision plus kit (Dako A/S). Nuclei were counterstained with haematoxylin before mounting. An example of the staining is shown in Figure 1 (Figure 1B). A positive and negative control slide was included in each IHC run; negative controls were incubated with an isotype matched control antibody at a concentration of $1 \mathrm{mg} \mathrm{ml}^{-1}$. Cell pellets known to express or not express PTEN were also included in each run. Antibody specificity was confirmed by western blot analysis (Figure 1C).

Staining was scored blind by two independent observers using a weighted histoscore method (Kirkegaard et al, 2006) also known as the H-score system (McCarty et al, 1986). Histoscores were calculated from the sum of $(1 \times$ the percentage of cells staining weakly positive $)+(2 \times$ the percentage of cells staining moderately positive $)+(3 \times$ the percentage of cells staining strongly positive) with a maximum histoscore of 300 . The interclass correlation coefficient (ICCC) between each observer was confirmed to measure consistency. The ICCC value was $>0.7$, which is classed as excellent as; an ICCC of 1 indicates identical score (Kirkegaard et al, 2006). The mean of the two observer's histoscores was used for the analysis. Changes in proteinexpression staining between hormone-sensitive and hormonerefractory cases were defined as an increase or decrease with the $95 \%$ confidence interval for the difference in the interobserver variation (ie, the mean difference between the histoscore that each observer assigns for protein expression \pm 2 s.d.; Edwards et al, 2003).

All statistical analyses were performed using the SPSS version 9.0 for Windows. Protein expression data were not normally distributed and is given as median and interquartile ranges. Wilcoxon signed-rank tests were used to compare protein expression between hormone-sensitive and hormone-refractory prostate cancer tumours. Survival analysis was conducted using the Kaplan-Meier method, and curves were compared with the log-rank test. Multivariate survival analysis and hazard ratios (HRs) were calculated using Cox regression analysis. A value of $P<0.05$ was considered statistically significant.

\section{RESULTS}

Patients in this cohort were diagnosed with locally advanced (50) or metastatic prostate cancer (18), and subsequently received surgery and androgen deprivation therapy (26 subcapsular bilateral orchidectomy, $44 \mathrm{GnRH}$ analogue and two had both). In all, 45 of the 68 patients also received an antiandrogen therapy and this included all those who received GnRH analogues. At initial diagnosis, the median age was $70(66-74)$ years and $26 \%$ of patients had metastatic disease. The median time to biochemical relapse was $2.32(1.48-4.00)$ years and the percentage of patients with metastatic disease had increased to $57 \%$. Sixty-one patients (89.7\%) died during follow-up, and median survival for these patients was $4.34(2.94-6.63)$ years. Seven patients were alive at the last follow-up; the median time of follow-up for all 68 patients was $4.34(2.86-6.74)$ years. 

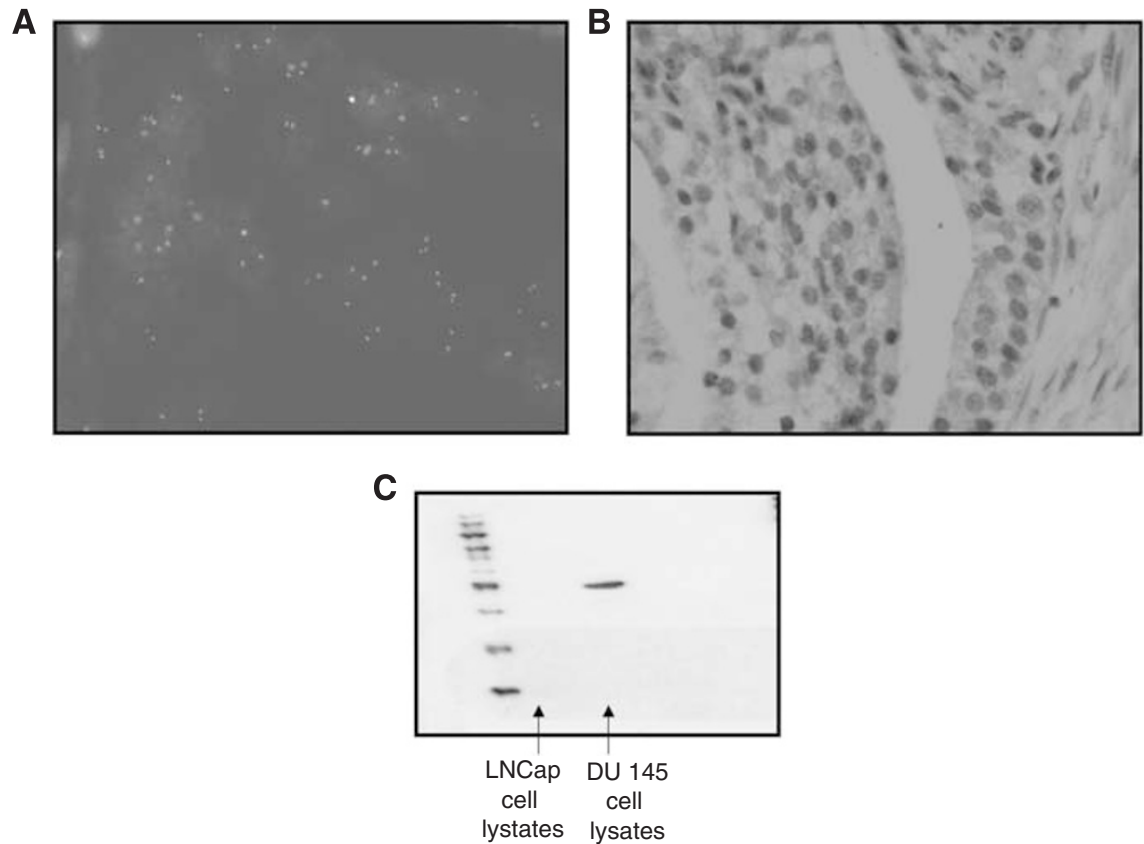

Figure I (A) shows an example of fluorescent in situ hybridisation for chromosome 10 (red signal) and PTEN (green signal). (B) Shows an example of immunohistochemistry for PTEN protein expression; in hormone-sensitive prostate cancer, both cytoplasmic expression and nuclear expression are present. (C) Western blot analysis for PTEN protein to confirm antibody specificity. LNCaP cells do not express PTEN protein, whereas DUI45 cells do express PTEN protein. Lane I, hormone-sensitive LNCaP cell lysates; lane 2, hormone-refractory LNCaP cell lysates; lane 3, DUI45 cell lysates.

Factors associated with time to biochemical relapse, time to death from biochemical relapse and disease-specific survival

When serum PSA level, age, metastasis and Gleason grade at diagnosis were analysed for this patient, cohort using the Kaplan Meier log-rank method, PSA at diagnosis $(P=0.036)$ and Gleason score at diagnosis $(P=0.010)$ were associated with shorter time to biochemical relapse.

Death at the time of biochemical relapse was associated with PSA level at relapse $(P=0.016)$. Overall survival was associated with the presence of metastases at relapse $(P=0.0019)$ and Gleason score at diagnosis $(P=0.049)$.

\section{Fluorescent in situ hybridisation}

PTEN deletions of the 68 prostate carcinomas investigated, PTEN/chromosome 10 ratio was successfully evaluated in 57 $(84 \%)$ cases. The remaining cases were excluded from the study because of insufficient tumour material in the cores. The mean PTEN/chromosome 10 ratio for the hormone-sensitive and hormone-refractory tumours was 0.98 (range: $0.71-1.11$ ) and 0.92 (range: $0.39-2.16$ ), respectively. Gene deletion as identified by FISH was observed in $23 \%$ of hormone-sensitive tumours; this increased significantly to $52 \%$ in hormone-refractory tumours $(P=0.044)$. Loss of one copy of PTEN was commonly observed, and this was heterogeneous in nature, being frequently observed in only one area of tumour. Loss of PTEN was correlated with prostate cancer progression; however, no correlation was observed between the loss of PTEN and Gleason score at diagnosis, loss of $P T E N$ and presence of metastasis at diagnosis or the loss of PTEN and PSA at diagnosis. When loss of PTEN was correlated with survival, a trend between the loss of PTEN and poorer diseasespecific survival was noted $(P=0.086)$; this was not independently significant by Cox regression analysis.
Gene number and chromosomal aneusomy In the informative cases, the mean PTEN gene copy number per counted cancer cell for the hormone-sensitive and hormone-refractory cases was 1.90 (range: $1.4-2.5$ ) and 1.71 (range: $0.80-3.45$ ) respectively. When assessing the frequency of chromosome aneusomy, the mean chromosomal copy number of chromosome 10 for the hormone-sensitive and hormone-refractory tumours was 1.93 (range: 1.75-2.25) and 1.87 (range: 1.60-2.05), respectively. Normal range for chromosomal copy number is $1.35-2.01$, in this cohort, none of the hormone-sensitive or hormone-refractory tumours appeared to have lost chromosome 10 as $0 \%$ had chromosome 10 copy number per counted cancer cell of less than 1.35 , which is the lower limit of the normal range. In contrast, $12 \%$ of the hormone-sensitive tumours and $21 \%$ of the hormonerefractory tumours had chromosome 10 copy numbers per counted cancer cell higher than that of the normal range. It was noted that this was a different subgroup of patients exhibiting the loss of PTEN gene.

\section{Immunohistochemistry}

Membrane, cytoplasmic and nuclear PTEN expression was observed in prostate tissue. In the hormone-sensitive tumours, membrane and nuclear expression were less frequently observed than cytoplasmic expression; 41 and $46 \%$ of patients, respectively, had membrane and nuclear expression compared with 95\% expressing PTEN in the cytoplasm. The interquartile range of expression for each location is shown in Table 1. This rate of expression did not significantly change in the hormonerefractory tissue. When median protein expression levels in the hormone-sensitive and hormone-refractory tissue were compared, no statistically significant change was observed at any cellular location. Loss of PTEN expression was heterogeneous. 
Table I Histoscore variation and comparison of staining intensity for hormone-sensitive and hormone-refractory tumours

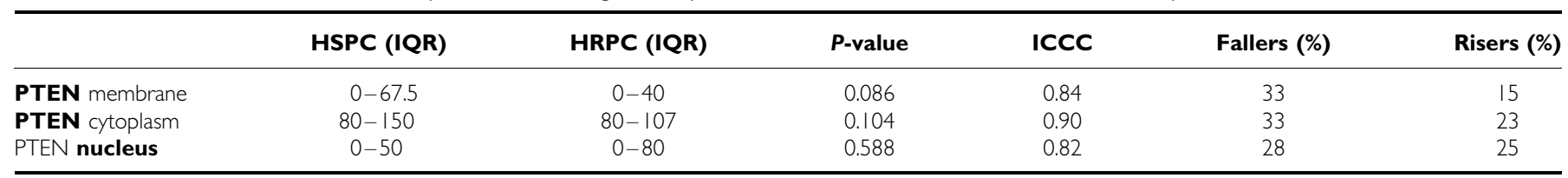

The interquartile range (IQR) for hormone-sensitive tumours (HSPC) and hormone-refractory tumours (HRPC) are shown. The $P$-value was compared using a Wilcoxon signedrank test. The interclass correlation coefficient (ICCC), which measures the consistency among observers for each protein, is consistently higher than 0.7, which is classed as excellent. The percentage of tumours that was defined as having a rise or fall in protein expression (calculated using the number of histoscore units that is defined as a change in expression) is also shown.

A

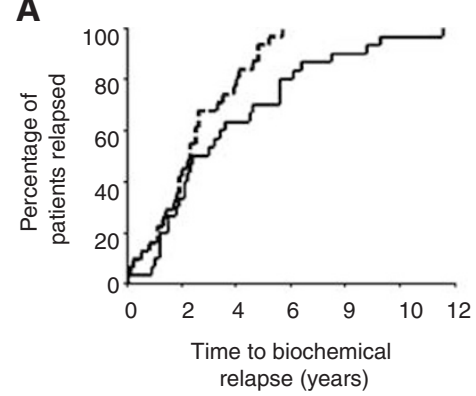

D

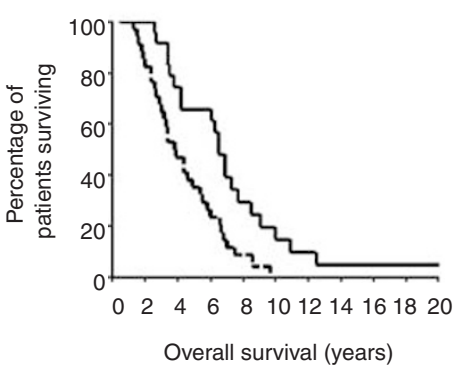

B

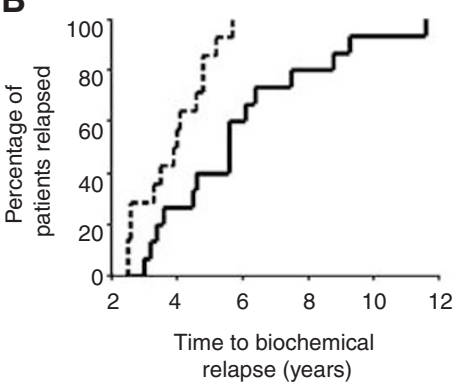

E

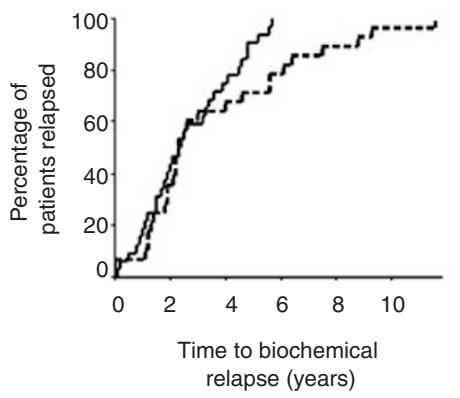

C

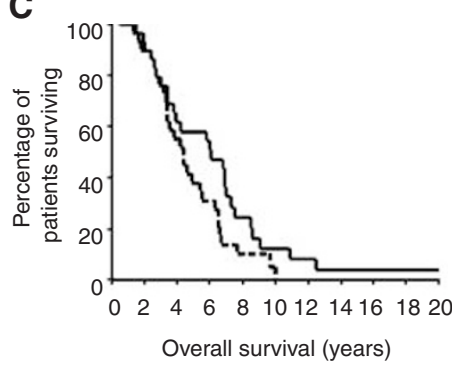

$\mathbf{F}$

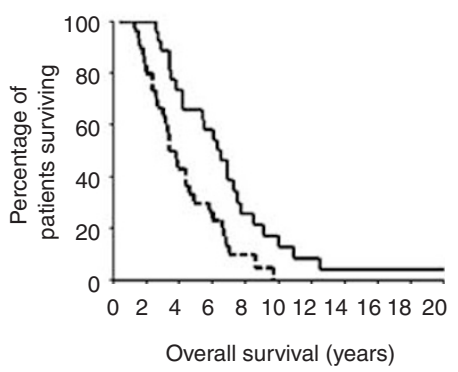

Figure 2 (A) Shows a Kaplan-Meier plot for high (above the median, solid line) and low (below the median, dotted line) PTEN cytoplasmic expression and time to biochemical relapse $(P=0.027)$. (B) Shows a Kaplan-Meier plot for high (above the median, solid line) and low (below the median, dotted line) PTEN cytoplasmic expression for patients that took longer than 30 months to relapse $(P=0.0035)$. ( $\mathbf{C})$ shows a Kaplan-Meier plot for high (above the median, solid line) and low (below the median, dotted line) PTEN cytoplasmic expression and disease-specific survival (labelled overall survival; $P=0.072$ ). (D) Shows a Kaplan-Meier plot for patients with tumours that have membrane PTEN expression (solid line) compared to patients whose tumours do not have nuclear PTEN expression (dotted line) and disease-specific survival (labelled overall survival; $P=0.002$ ). (E) Shows a Kaplan-Meier plot for patients with tumours that have nuclear PTEN expression (dotted line) compared to patients whose tumours do not have nuclear PTEN expression (solid line) and time to biochemical relapse $(P=0.078)$. (F) Shows a Kaplan-Meier plot for patients with tumours that have nuclear PTEN expression (solid line) compared to patients whose tumours do not have nuclear PTEN expression (dotted line) and disease-specific survival (labelled overall survival; $P=0.003$ ).

\section{Hormone-sensitive tumours}

To determine whether protein expression was linked to time to biochemical relapse, Kaplan-Meier graphs were plotted for the hormone-sensitive tumours expressing low levels of protein $(<$ median histoscore) $v s$ high levels of protein ( $>$ median histoscore), and compared using the log-rank test. The patients whose tumours expressed low levels of PTEN in the cytoplasm were shown to have relapsed significantly earlier than those patients whose tumours expressed high levels of PTEN in the cytoplasm (Figure $2 \mathrm{~A}, P=0.027$ ). Cox regression analysis indicates that cytoplasmic PTEN expression is independent of known clinical prognostic factors $(P=0.028$, HR $0.51 \quad(95 \%$ CI $0.27-$ $0.93)$. It was noted, however, that the Kaplan-Meier curves did not separate until approximately 2.5 years after diagnosis. Therefore, PTEN loss appeared to be influencing relapse in those patients who took more than 30 months to relapse. If patients who relapsed within 30 months were excluded from the analysis, the median time to relapse for those with low PTEN expression was 3.9 (IQR 2.98 - 4.92) years compared to $5.6(4.36-6.84)$ years for those with high PTEN expression $(P=0.0035$; Figure $2 \mathrm{~B})$. In addition, those patients with high levels of cytoplasmic PTEN expression in their hormone-sensitive tumours were observed to have longer median overall survival (6.1 years (IQR 2.8-9.4)) compared to those with low PTEN expression (4.4 years (IQR 3.3 - 5.4)), although this did not reach significance $(P=0.072$; Figure $2 \mathrm{C})$. Again the curves first separate approximately 30 months after diagnosis.

Expression of phosphorylated Akt at serine 473 (activated Akt) for this cohort of patients had already been established in a previous study (McCall et al, 2008). Levels of phosphorylated Akt expression in tumours that expressed low levels of PTEN was higher compared to tumours that expressed high levels of PTEN $(P=0.047)$.

The median for both PTEN membrane and nuclear expression in the hormone-sensitive tumours was 0 ; therefore patients were divided into those patients whose tumours did not express PTEN 
at these locations and those that did. Patients with PTEN membrane expression in their hormone-sensitive tumour had significantly longer overall survival than those patients without (Figure $2 \mathrm{D}, P=0.002$ ). The median time to relapse for patients whose tumours did not express PTEN in the membrane was 3.8 years (IQR 2.7-4.85) compared to 6.5 (IQR 5.8-7.2) years for patients with membrane expression.

Patients with no nuclear PTEN expression relapsed earlier than those with nuclear PTEN expression, although this did not reach significance (Figure 2E, $P=0.078$ ). PTEN nuclear expression was associated with overall survival, those patients whose tumours had no nuclear PTEN expression had a significantly shorter overall survival compared to those patients with PTEN nuclear expression (Figure $2 \mathrm{~F}, P=0.003$ ). Median overall survival was 3.4 years (IQR 2.6-4.2) compared to 6.5 years (IQR 5.1-7.8) that confers a survival advantage of 3 years for those patients whose tumours express nuclear PTEN. Nuclear PTEN expression was demonstrated to be an independent prognostic marker by Cox regression analysis, when compared with known clinical prognostic parameters $(P=0.031$, HR 0.52 (95\% CI $0.29-0.95)$.

As observed with membrane expression, phosphorylated Akt expression was lower in the nucleus of tumours with high levels of nuclear PTEN than those with low levels, but this did not reach significance $(P=0.132)$. In contrast, PTEN membrane expression correlated strongly with nuclear PTEN expression $(P<0.001$, $\left.R_{\mathrm{S}} 0.66\right)$.

\section{Hormone-refractory tumours}

Phosphatase and tensin homologue deleted on chromosome 10 expression levels in the hormone-refractory tumours were not associated with time to death from relapse, disease-specific death from relapse or with the presence of metastasis.

\section{DISCUSSION}

The PTEN tumour suppressor has emerged as a critical regulator of cellular processes, which is frequently mutated or deleted in a number of human cancers, including prostate cancer. Fluorescent in situ hybridisation and IHC have demonstrated that a significant proportion of patients have heterogeneous PTEN deletion and loss of PTEN protein expression, which is, associated with clinical outcome measures.

The frequency and mode of PTEN inactivation reported at various stages of clinical prostate cancer are variable (Verhagen et al, 2006). In this study, we investigated the level of PTEN loss by FISH in matched hormone-sensitive and hormone-refractory tumours. Loss was noted in $23 \%$ hormone-sensitive tumours compared with $52 \%$ hormone-refractory tumours; these rates are similar to those previously reported by FISH analysis (Verhagen et al, 2006). Fluorescent in situ hybridisation depending on the region that the probe binds to does not always detect small deletions, and in the case of our study, the probe covers the whole of the PTEN gene. Therefore, in this study, loss of the whole gene is being measured. It was observed that very few tumours had homogeneous PTEN deletion and complete loss of PTEN expression (2\%), but almost all have heterogeneous loss of expression; this is consistent with previous reports (Verhagen et al, 2006). In this study, PTEN loss does not correlate with PTEN gene deletion, although all tumours with PTEN deletion have low PTEN expression. An explanation for low PTEN expression in tumours that appear not to have PTEN deletion is the hypermethylation of the PTEN promoter region. Evidence for promoter hypermethylation has been reported in prostate cancer xenografts (Whang et al, 1998). Although the mechanism of PTEN inactivation is currently controversial and possibly because of different mechanisms in different tumours, it is widely accepted that PTEN loss is one of the most common events associated with prostate cancer (Majumder and Sellers, 2005). Consistent with these findings, this study observed a higher rate of PTEN deletion by FISH in hormonerefractory compared to hormone sensitive tumours, suggesting that PTEN loss is associated with tumour progression. Although this was not significantly associated with clinical outcome measures, a trend was observed demonstrating that those patients with PTEN loss had shorter overall survival. If the FISH studies were expanded to a larger data set, these results might have reached significance.

As predicted, loss or low cytoplasmic PTEN expression was independently associated with time to relapse and linked with increased Akt activation. Surprisingly, however, this was observed to be a late event with curves separating 30 months following diagnosis, suggesting that other factors, such as PI3K expression, may also contribute to Akt activation and disease progression. Cytoplasmic PTEN expression was only weakly associated with overall survival, and this did not reach significance in this study.

However, in addition to cytoplasmic PTEN expression, nuclear PTEN expression was also observed. Unlike cytoplasmic PTEN expression, loss of nuclear PTEN expression was weakly associated with time to relapse, and this did not reach significance. Nuclear PTEN expression was, however, independently strongly associated with overall survival, and the curves on the Kaplan-Meier plot begun to separate almost immediately after diagnosis. These results in combination with the lack of correlation with Akt activation suggest that the role of PTEN in the nucleus is independent of cytoplasmic PTEN. It is now recognised that PTEN has a function in the nucleus, and reports of PTEN nuclear localisation have begun to multiply over the past few years in tumour and non-tumour cells (Gimm et al, 2000). Chung et al (2005) have demonstrated that PTEN has dual nuclear localisation signal-like sequences that mediate nuclear import, and have shown that nuclear PTEN is required for PTEN-mediated cell-cycle arrest and growth inhibition through the downregulation of cyclin D1. Reports from the literature also suggest that PTEN localises to the nucleus during the $G_{0}-G_{1}$ phase of cell cycle and mediates growth suppression through the inhibition of MAP kinase phosphorylation independent of Akt activation (Chung and Eng, 2005; Chung et al, 2006). Results from our study support the hypothesis that PTEN has a distinct function in the nucleus independent of its cytoplasmic role.

However, it is a possibility that nuclear PTEN is simply a surrogate marker of PTEN activation as in vitro studies demonstrate that following phosphorylation, PTEN is released from the membrane-bound scaffolding proteins and enters the nucleus. In support of this, we report a correlation between membrane and nuclear PTEN expression $(P<0.001$, Rs 0.66$)$, and PTEN membrane expression is also linked to survival. However, in contrast to cytoplasmic PTEN expression, no correlations were observed between nuclear PTEN expression and Akt activation; therefore, the evidence to support nuclear PTEN as a surrogate marker of PTEN cytoplasmic activation is not convincing in this study.

In summary, the rate of loss of PTEN as measured by FISH increased with disease progression, and a trend was noted between $P T E N$ loss and poorer disease-specific survival, suggesting that this arm of the study should be expanded to larger cohort. In addition, both cytoplasmic and nuclear PTEN are independently associated with good outcome measures in hormone-sensitive prostate cancer, but appear to have independent roles.

\section{ACKNOWLEDGEMENTS}

I would like to acknowledge AICR and Glasgow Royal Infirmary Endowment Funds for funding this project. 


\section{REFERENCES}

Arnold JT, Isaacs JT (2002) Mechanisms involved in the progression of androgen-independent prostate cancers: it is not only the cancer cell's fault. Endocr Relat Cancer 9: 61-73

Bookstein R, Rio P, Madreperla SA, Hong F, Allred C, Grizzle WE, Lee WH (1990) Promoter deletion and loss of retinoblastoma gene expression in human prostate carcinoma. Proc Natl Acad Sci USA 87: $7762-7766$

Burton JL, Oakley N, Anderson JB (2000) Recent advances in the histopathology and molecular biology of prostate cancer. BJU Int 85: $87-94$

Cancer Research UK web site (2004) Cancer Research UK web site. Cancer Stats. Mortality: UK. Available at http://www.cancerresearchuk.org

Chung JH, Eng C (2005) Nuclear-cytoplasmic partitioning of phosphatase and tensin homologue deleted on chromosome 10 (PTEN) differentially regulates the cell cycle and apoptosis. Cancer Res 65: 8096-8100

Chung JH, Ginn-Pease ME, Eng C (2005) Phosphatase and tensin homologue deleted on chromosome 10 (PTEN) has nuclear localization signal-like sequences for nuclear import mediated by major vault protein. Cancer Res 65: 4108-4116

Chung JH, Ostrowski MC, Romigh T, Minaguchi T, Waite KA, Eng C (2006) The ERK1/2 pathway modulates nuclear PTEN-mediated cell cycle arrest by cyclin D1 transcriptional regulation. Hum Mol Genet 15: $2553-2559$

Davies MA, Koul D, Dhesi H, Berman R, McDonnell TJ, McConkey D, Yung WK, Steck PA (1999) Regulation of Akt/PKB activity, cellular growth, and apoptosis in prostate carcinoma cells by MMAC/PTEN. Cancer Res 59: $2551-2556$

Edwards J, Krishna NS, Grigor KM, Bartlett JM (2003) Androgen receptor gene amplification and protein expression in hormone refractory prostate cancer. Br J Cancer 89: $552-556$

Eng C (2003) PTEN: one gene, many syndromes. Hum Mutat 22: $183-198$

Feilotter HE, Nagai MA, Boag AH, Eng C, Mulligan LM (1998) Analysis of PTEN and the $10 \mathrm{q} 23$ region in primary prostate carcinomas. Oncogene 16: $1743-1748$

Fenci I, Woenckhaus J (2002) The tumour suppressor PTEN and the cell cycle inhibitor p27(KIP1) in prostate carcinoma and prostatic intraepithelial neoplasia (PIN) - an immunohistochemical study. Br J Cancer 86: $\mathrm{S} 79-\mathrm{S} 80$

Gimm O, Perren A, Weng LP, Marsh DJ, Yeh JJ, Ziebold U, Gil E, Hinze R, Delbridge L, Lees JA, Mutter GL, Robinson BG, Komminoth P, Dralle H, Eng C (2000) Differential nuclear and cytoplasmic expression of PTEN in normal thyroid tissue, and benign and malignant epithelial thyroid tumors. Am J Pathol 156: $1693-1700$

Gray IC, Stewart LM, Phillips SM, Hamilton JA, Gray NE, Watson GJ, Spurr NK, Snary D (1998) Mutation and expression analysis of the putative prostate tumour- suppressor gene PTEN. Br J Cancer 78: 300

He WW, Sciavolino PJ, Wing J, Augustus M, Hudson P, Meissner PS, Curtis RT, Shell BK, Bostwick DG, Tindall DJ, Gelmann EP, AbateShen C, Carter KC (1997) A novel human prostate-specific, androgen-regulated homeobox gene (NKX3.1) that maps to 8 p21, a region frequently deleted in prostate cancer. Genomics 43: 69-77

Isaacs W, Kainu T (2001) Oncogenes and tumor suppressor genes in prostate cancer. Epidemiol Rev 23: $36-41$

Johnson MI, Hamdy FC (1998) Apoptosis regulating genes in prostate cancer (review). Oncol Rep 5: 553-557
Karan D, Lin MF, Johansson SL, Batra SK (2003) Current status of the molecular genetics of human prostatic adenocarcinomas. Int J Cancer 103: $285-293$

Kirkegaard T, Edwards J, Tovey S, McGlynn LM, Krishna SN, Mukherjee R, Tam L, Munro AF, Dunne B, Bartlett JM (2006) Observer variation in immunohistochemical analysis of protein expression, time for a change? Histopathology 48: $787-794$

Lian ZL, Di Cristofano A (2005) Class reunion: PTEN joins the nuclear crew. Oncogene 24: 7394-7400

Majumder PK, Sellers WR (2005) Akt-regulated pathways in prostate cancer. Oncogene 24: 7465-7474

McCall P, Gemmell LK, Mukherjee R, Bartlett JMS, Edwards J (2008) Phosphorylation of the androgen receptor is associated with reduced survival in hormone refractory prostate cancer patients. $\mathrm{Br} J$ Cancer 98: $1094-1101$

McCarty Jr KS, Szabo E, Flowers JL, Cox EB, Leight GS, Miller L, Konrath J, Soper JT, Budwit DA, Creasman WT (1986) Use of a monoclonal antiestrogen receptor antibody in the immunohistochemical evaluation of human tumors. Cancer Res 46: 4244s-4248s

McMenamin ME, Soung P, Perera S, Kaplan I, Loda M, Sellers WR (1999) Loss of PTEN expression in paraffin-embedded primary prostate cancer correlates with high Gleason score and advanced stage. Cancer Res 59: $4291-4296$

Murillo H, Huang H, Schmidt LJ, Smith DI, Tindall DJ (2001) Role of PI3K Signaling in Survival and Progression of LNCaP Prostate Cancer Cells to the Androgen Refractory State. Endocrinology 142: $4795-4805$

Myers MP, Pass I, Batty IH, Van der Kaay J, Stolarov JP, Hemmings BA, Wigler MH, Downes CP, Tonks NK (1998) The lipid phosphatase activity of PTEN is critical for its tumor suppressor function. Proc Natl Acad Sci USA 95: $13513-13518$

Phillips SM, Barton CM, Lee SJ, Morton DG, Wallace DM, Lemoine NR, Neoptolemos JP (1994) Loss of the retinoblastoma susceptibility gene (RB1) is a frequent and early event in prostatic tumorigenesis. $\mathrm{Br} J$ Cancer 70: $1252-1257$

Reiss K, Wang JY, Romano G, Furnari FB, Cavenee WK, Morrione A, Tu X, Baserga R (2000) IGF-I receptor signaling in a prostatic cancer cell line with a PTEN mutation. Oncogene 19: 2687-2694

Suzuki H, Freije D, Nusskern DR, Okami K, Cairns P, Sidransky D, Isaacs WB, Bova GS (1998) Interfocal heterogeneity of PTEN/MMAC1 gene alterations in multiple metastatic prostate cancer tissues. Cancer Res 58: 204-209

Verhagen P, van Duijn P, Hermans K, Looijenga L, van Gurp R, Stoop H, van der KT, Trapman J (2006) The PTEN gene in locally progressive prostate cancer is preferentially inactivated by bi-allelic gene deletion. J Pathol 208: 699-707

Wang SI, Parsons R, Ittmann M (1998) Homozygous deletion of the PTEN tumor suppressor gene in a subset of prostate adenocarcinomas. Clin Cancer Res 4: $811-815$

Watters AD, Stacey MW, Going JJ, Grigor KM, Cooke TG, Sim E, Barlett JMS (1998) Genetic alterations of N-acetyl transferase in transitional cell carcinoma of the bladder. Br J Cancer 78: $154-156$

Whang YE, Wu X, Suzuki H, Reiter RE, Tran C, Vessella RL, Said JW, Isaacs WB, Sawyers CL (1998) Inactivation of the tumor suppressor PTEN/ MMAC1 in advanced human prostate cancer through loss of expression. Proc Natl Acad Sci USA 95: 5246-5250 